(C) OPEN ACCESS
- Additional material is published online only. To view please visit the journal online (http://dx.doi.org/10.1136/ thoraxjnl-2018-211897).

${ }^{1}$ Laboratory of Molecular Angiogenesis, GIGA-R, University of Liège, Liège, Belgium

${ }^{2}$ Pneumology Department, CHU Liège, Liège, Belgium ${ }^{3}$ Laboratory of Pneumology, GIGA-I3, University of Liège, Liège, Belgium

${ }^{4}$ Laboratoire de Biologie Cellulaire et Tissulaire, GIGA-R, University of Liège, Liège, Belgium

5 Laboratoire de Signalisation et Interactions des Protéines, GIGA-R, University of Liège, Liège, Belgium

\section{Correspondence to}

Dr Makon-Sébastien Njock, Laboratory of Molecular Angiogenesis, Université de Liège - GIGA centre, Liège, 4000, Belgium:

sebastien.njock@gmail.com

M-SN and JG contributed equally.

Received 4 April 2018 Revised 24 August 2018 Accepted 27 August 2018 Published Online First 22 September 2018
Check for updates

(C) Author(s) (or their employer(s)) 2019. Re-use permitted under CC BY-NC. No commercial re-use. See rights and permissions. Published by BMJ.

To cite: Njock M-S, Guiot J, Henket MA, et al. Thorax 2019:74:309-312.

\title{
Sputum exosomes: promising biomarkers for idiopathic pulmonary fibrosis
}

\author{
Makon-Sébastien Njock, ${ }^{1}$ Julien Guiot, ${ }^{2,3}$ Monique A Henket, ${ }^{2,3}$ Olivier Nivelles, ${ }^{1}$ \\ Marc Thiry, ${ }^{4}$ Franck Dequiedt, ${ }^{5}$ Jean-Louis Corhay, ${ }^{2,3}$ Renaud E Louis, ${ }^{2,3}$ \\ Ingrid Struman ${ }^{1}$
}

\begin{abstract}
Idiopathic pulmonary fibrosis (IPF) is a progressive fibrosing interstitial lung disease of unknown aetiology which leads rapidly to death. As diagnosis of IPF is complex, we aimed to characterise microRNA (miRNA) content of exosomes from sputum of patients with IPF. Using miRNA quantitative PCR array, we found a substantial dysregulation of sputum exosomal miRNA levels between patients with IPF and healthy subjects and identified a unique signature of three miRNAs. Interestingly, we found a negative correlation
\end{abstract}
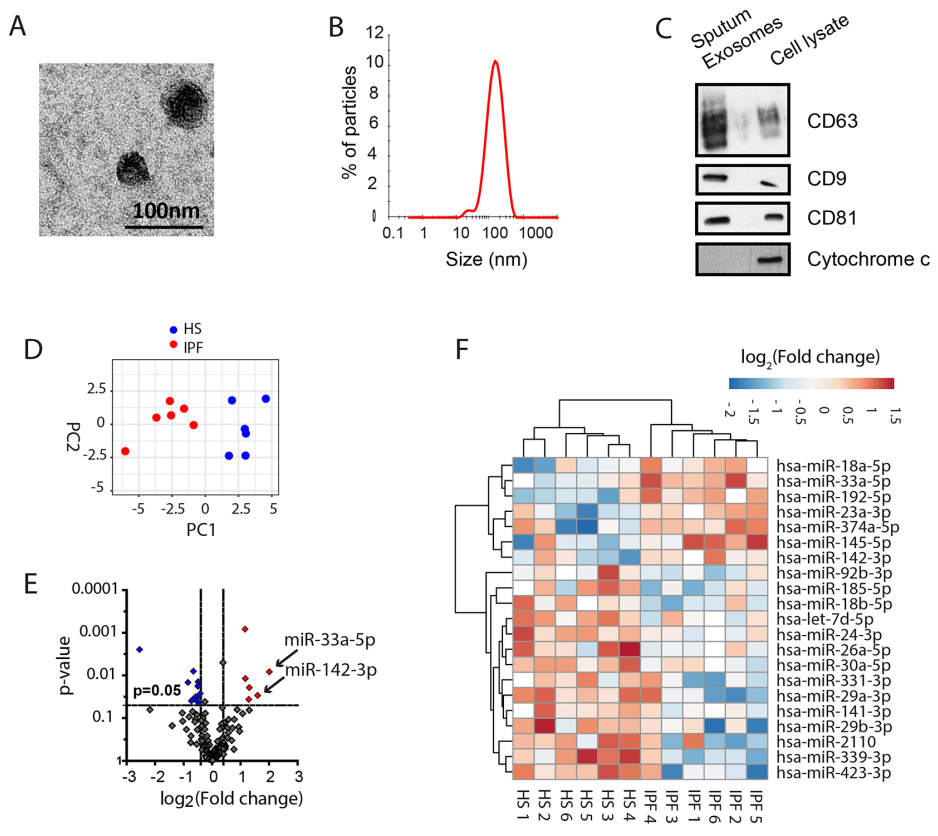

G

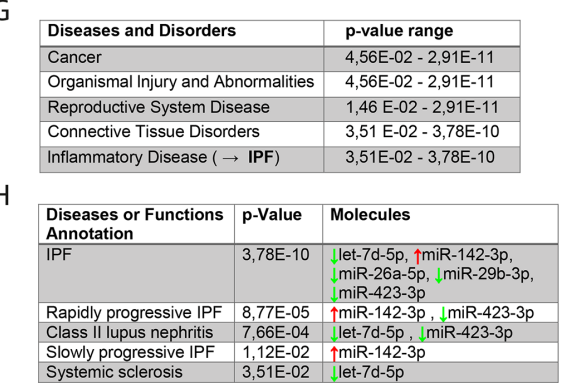

Figure 1 Identification of a subset of exosomal microRNAs (miRNAs) dysregulated in the sputum of patients with idiopathic pulmonary fibrosis (IPF). Characterisation of exosome-like vesicles released from sputum by (A) transmission electron microscopy, (B) dynamic light scattering analysis $(140 \pm 19.1 \mathrm{~nm}, \mathrm{n}=3)$ and $(C)$ western blotting. Presence of exosomal markers, CD63, CD9, CD81 and mitochondrial protein cytochrome $c$ in lysates from sputum-derived exosomes and cell lysate. (D) Principal component analysis plot on miRNA expression data from sputum-derived exosomes from healthy subjects (HS) and patients with IPF. (E) Volcano plot and (F) heat map of differentially expressed miRNAs across sputum-derived exosomes from HS and patients with IPF (fold change $>1.3$ and $p<0.05$ ). (G) Diseases and disorders or (H) functions annotation associated with exosomal miRNAs dysregulated in the sputum of patients with IPF predicted by ingenuity pathway analysis. 
aetiology which leads rapidly to death. ${ }^{1}$ As diagnosis of IPF is complex, the search for novel biomarkers is a central challenge for the future of translational research. ${ }^{2}$ Induced sputum has been previously identified as a potential biofluid of interest in $\mathrm{IPF}^{3}$ A growing list of studies reported the presence of a specific type of extracellular vesicles, exosomes, in several biofluids (including blood, bronchoalveolar lavage fluid, saliva) and an alteration of their composition during the course of diseases, ${ }^{4-6}$ highlighting the value of these vesicles as a new source of biomarkers. Most of the studies performed so far have searched for exosomal markers in sera or plasma samples, while only one recent study reported the presence of exosomes in the sputum of patients with asthma. ${ }^{7}$ The aim of our study was to characterise microRNA (miRNA) content of sputum exosomes of patients with IPF versus healthy subjects (HS) in order to identify novel diagnostic biomarkers.

\section{METHODS}

For full technical details, see the online Supplementary data. Patients with IPF were recruited from the ambulatory care polyclinics of both Liège University. All subjects gave written consent before their enrolment. The patient characteristics are summarised in the online Supplementary table S1. After collecting the induced sputum, exosomes were isolated from the sputum supernatant by using a standard ultracentrifugation protocol.

\section{RESULTS}

The isolated vesicles present a spherical morphology (figure 1A), an average size distribution of $140 \mathrm{~nm}( \pm 19.1 \mathrm{~nm})$ (figure 1B), an enrichment of several exosomal markers, CD63, CD9 and CD81 and an absence of mitochondrial cytochrome C (figure 1C), which clearly indicate that this vesicle population is enriched in exosomes. The miRNA expression profiling of sputum-derived exosomes from six patients with IPF and six HS by miRNA quantitative PCR arrays allowed us to identified 21 miRNAs differentially expressed, among which seven miRNAs were upregulated and 14 miRNAs were downregulated in patients with IPF (figure 1D-F and online Supplementary table S2). In order to assess whether some dysregulated miRNAs correlate with the pathophysiology of the disease, we subjected a list of seven miRNAs with aberrant expression (three upregulated miRNAs: miR-33a-5p, miR-142-3 $p$ and miR-192-5 p; four downregulated miRNAs: let-7d-5p, miR-26a-5p, miR-29b-3p and miR-423-3p) to ingenuity pathway analysis tool. The results indicated that these miRNAs are involved in the development of inflammatory diseases, among which the IPF disease is the most relevant one $(p=3.78 E-10)$ (figure $1 G, H)$. The functional analysis of
A

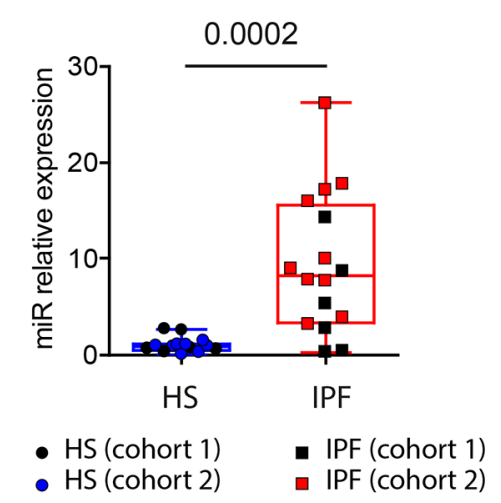

B

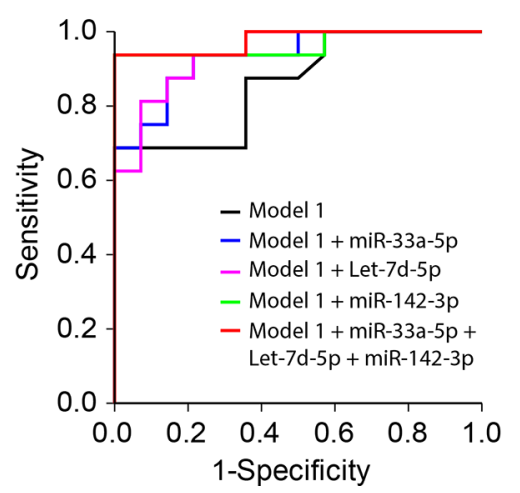

$\operatorname{miR}-33 a-5 p$

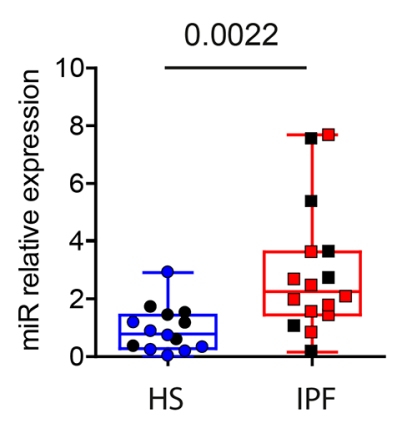

C

\begin{tabular}{lllll}
\hline \multicolumn{5}{c}{ IPF vs HS } \\
\cline { 2 - 5 } & AUC & $95 \% \mathrm{Cl}$ & $\begin{array}{l}\text { \% sensitivity } \\
\text { at 80\% specificity }\end{array}$ & p-value \\
\hline Model 1 & 0.864 & $0.735-0.993$ & 68.7 & $<0.001$ \\
Model 1 + miR-33a-5p & 0.933 & $0.848-1.000$ & 87.5 & $<0.0001$ \\
Model 1 + Let-7d-5p & 0.942 & $0.865-1.000$ & 87.5 & $<0.0001$ \\
Model 1 + miR-142-3p & 0.964 & $0.893-1.000$ & 93.75 & $<0.0001$ \\
Model 1 + miR-33a-5p+ & 0.978 & $0.930-1.000$ & 93.5 & $<0.0001$ \\
Let-7d-5p + miR-142-3p & & & &
\end{tabular}

Figure 2 Diagnostic value of sputum-derived exosomal miR-142-3p, miR-33a-5p and let-7d-5p for idiopathic pulmonary fibrosis (IPF) disease. (A) Quantitative real-time PCR analysis of differentially expressed microRNAs (miRNAs) in sputum-derived exosomes from patients with IPF ( $\mathrm{n}=16$ ) compared with healthy subjects (HS; $n=14$ ). Cohort 1 was used to identify exosomal miRNAs dysregulated in the sputum of patients with IPF; cohort 2 is the validation cohort. (B) Receiver operator characteristic (ROC) curves with (C) corresponding area under the curves (AUCs) for comparing the ability of miR-142-3p, miR-33a-5p and let-7d-5p to discriminate patients with IPF versus HS. ROC curves were constructed by combining the two cohorts (cohort 1 and cohort 2). Five logistic regression models were generated: (1) model 1: age and sex; (2) model 2: model 1+miR-33a-5p; (3) model 3: model 1+let-7d-5p; (4) model 4: model 1+miR-142-3p and (5) model 5: model 1+miR-33a-5P+let-7d-5P+miR-142-5p. 
their predicted targets revealed that these regulators play key roles in several pathophysiological processes involved in the initiation and progression of IPF disease, such as deposition of extracellular matrix, collagen secretion and epithelial to mesenchymal transition (online Supplementary figure S1).

We further validated three promising biomarker candidates (miR-142-3p, miR-33a-5p, let-7d-5p) by quantitative realtime-PCR in an independent cohort (cohort 2) consisting of 10 patients with IPF and eight HS (online Supplementary figure S2). By combining the two cohorts, we observed significantly increased levels of miR-142-3p and miR-33a-5p (9.4 and 3.13 fold, respectively) and decreased levels of let-7d-5p (0.49-fold) in sputum-derived exosomes from patients with IPF $(n=16)$ compared with HS $(n=14)$ (figure 2A). The results were not altered after adjusting for age or gender distribution (online Supplementary tables S3 and S4).

To investigate their diagnostic value, we constructed receiver operator characteristic (ROC) curves using logistic regression models with adjustment for age and sex for each altered miRNAs (figure 2B). The area under the ROC curve (AUC) of the model 1 , comprising age and sex, was 0.864 (95\% CI 0.735 to 0.993 , $\mathrm{p}<0.001$ ) (figure $2 \mathrm{C}$ ). The addition of miR-33a-5p, let-7d-5p or miR-142-3p to the model increased the AUC to 0.933 (95\% CI 0.848 to $1.000, p<0.0001), 0.942(95 \%$ CI 0.865 to 1.000 , $\mathrm{p}<0.0001)$ and $0.964(95 \%$ CI 0.893 to $1.000, \mathrm{p}<0.0001)$, respectively (figure $2 \mathrm{C}$ ). When combining the three miRNAs, the AUC reaches 0.978 (95\% CI 0.930 to $1.000, \mathrm{p}<0.0001$ ) suggesting that this miRNA signature may be useful for IPF detection and diagnosis.

Then, the correlation between the expression level of sputum exosomal miRNAs and lung function was studied in the group of patients with IPF. Correlation studies revealed a negative correlation between diffusing capacity of the lungs for carbon monoxide/alveolarvolume (DLCO/VA) and miR-142-3p $(\mathrm{r}=-0.68, \mathrm{p}=0.010)$ and a positive one with let- $7 \mathrm{~d}-5 \mathrm{p}(\mathrm{r}=0.55$, $\mathrm{p}=0.048$ ) (figure 3 ). All these observations suggest that sputum exosomal miRNAs are associated with the severity of lung disease in the particular case of patients with IPF.

\section{DISCUSSION}

We identified for the first time miRNAs modifications from sputum-derived exosomes in patients with IPF as potential biomarkers correlated with disease severity. We propose a novel signature composed of three miRNAs (miR-142-3p, miR-33a-5p, let-7d-5p) to discriminate with a high specificity and sensibility patients with IPF of HS.

One of the main finding of our research is the negative correlation observed for miR-142-3 $p$ with lung function assessed by DLCO/VA, a marker of alveolo-capillar function, whereas let-7d-5p goes in the opposite direction. This observation suggests that those miRNAs are not only promising biomarkers but also putative players in the disease.

Interestingly, miR-142-3p was shown to contribute to the proper proliferation of mesenchymal progenitors by controlling WNT signalling. ${ }^{8}$ Elevated levels of miR-142-3p might thus lead to an altered balance between mesenchymal cell proliferation and differentiation, a feature observed during IPF progression. Furthermore, let-7d-5p downregulation has been widely reported as primordial miRNA involved IPF pathophysiology, by contributing to epithelial mesenchymal transition in lung epithelial cells. ${ }^{9}$ Of note, miR-33a-5p has been recently implicated in liver fibrosis. ${ }^{10}$ This observation deserves further investigations on the role of this miRNA in lung fibrosis.
Our study identified miRNAs previously identified in plasma as well as new miRNAs with potential functional role in IPF progression. Our findings may thus lead to a better understanding about the roles of identified sputum exosomal miRNAs
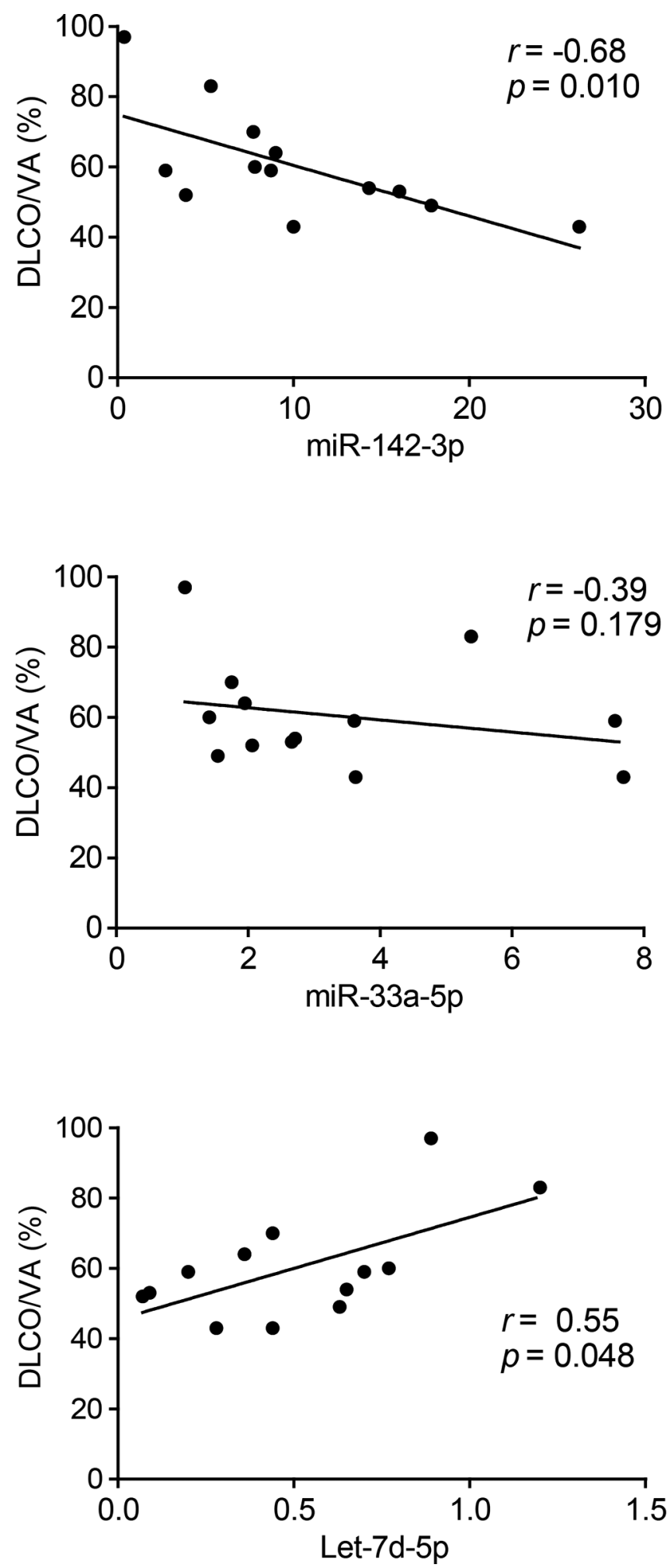

Figure 3 Correlation analysis of lung function and sputum-derived exosomal microRNAs (miRNAs) in patients with idiopathic pulmonary fibrosis (IPF). Correlation between diffusing capacity of the lungs for carbon monoxide (DLCO)/alveolar volume (VA) (\%pred) and miRNA levels in patients with IPF. 
in IPF pathogenesis and thus open new avenues for therapeutic approaches. Unfortunately, the cross-sectional design of our study does not allow the identification of predictive and/ or prospective biomarkers. Further longitudinal studies will be performed to validate the potential of sputum exosomal miRNAs as biomarkers in IPF to predict disease severity and evolution.

Acknowledgements We would like to thank the platform support staff from the GIGA Research Center. We gratefully thank Dr Laurence Seidel for her help with statistical analysis.

Contributors MSN and JG designed and performed experiments, analysed the data and wrote the manuscript. MAH collected and processed sputum samples. ON performed experiments. MT performed TEM study. FD, JLC and REL contributed to experiments analysis and interpretation of the results. IS design, analysed, supervised the experiment and wrote the manuscript.

Funding This study was supported by the Fonds d'Investissement de Recherché Scientifique du Centre Hospitalier Universitaire de Liège (FIRS); Centre Hospitalier Universitaire de Liège (CHU); the University of Liège (ULg); the Fonds National de la Recherche Scientifique (FNRS) and the fonds Léon Frédérica.

Competing interests None declared.

Patient consent Obatined.

Ethics approval The protocol was approved by the ethics committee of $\mathrm{CHU}$ of Liège (Belgian number: B707201422832; ref: 2014/302).

Provenance and peer review Not commissioned; externally peer reviewed.

Open access This is an open access article distributed in accordance with the Creative Commons Attribution Non Commercial (CC BY-NC 4.0) license, which permits others to distribute, remix, adapt, build upon this work non-commercially, and license their derivative works on different terms, provided the original work is properly cited, appropriate credit is given, any changes made indicated, and the use is non-commercial. See: http://creativecommons.org/licenses/by-nc/4.0/.

\section{REFERENCES}

1 Raghu G. Idiopathic pulmonary fibrosis: guidelines for diagnosis and clinical management have advanced from consensus-based in 2000 to evidence-based in 2011. Eur Respir J 2011;37:743-6.

2 Guiot J, Moermans $C$, Henket M, et al. Blood biomarkers in idiopathic pulmonary fibrosis. Lung 2017;195:273-80.

3 Guiot J, Henket M, Corhay JL, et al. Sputum biomarkers in IPF: evidence for raised gene expression and protein level of IGFBP-2, IL-8 and MMP-7. PLoS One 2017; 12:e0171344.

4 Gallo A, Tandon M, Alevizos I, et al. The majority of microRNAs detectable in serum and saliva is concentrated in exosomes. PLoS One 2012;7:e30679.

5 Admyre C, Grunewald J, Thyberg J, et al. Exosomes with major histocompatibility complex class II and co-stimulatory molecules are present in human BAL fluid. Eur Respir J 2003;22:578-83.

6 Njock MS, Fish JE. Endothelial miRNAs as cellular messengers in cardiometabolic diseases. Trends Endocrinol Metab 2017;28:237-46.

7 Sánchez-Vidaurre S, Eldh M, Larssen P, et al. RNA-containing exosomes in induced sputum of asthmatic patients. J Allergy Clin Immunol 2017;140:1459-61.

8 Carraro G, Shrestha A, Rostkovius J, et al. miR-142-3p balances proliferation and differentiation of mesenchymal cells during lung development. Development 2014;141:1272-81.

9 Pandit KV, Corcoran D, Yousef $\mathrm{H}$, et al. Inhibition and role of let-7d in idiopathic pulmonary fibrosis. Am J Respir Crit Care Med 2010;182:220-9.

10 Huang C-F, Sun C-C, Zhao F, et al. miR-33a, an important marker and putative therapeutic target in chronic HBV-induced fibrosis. RNA Dis 2015;1. 